\title{
Canonical Wnt signaling activates miR-34 expression during osteoblastic differentiation
}

\author{
MASATO TAMURA, MAKI UYAMA, YURI SUGIYAMA and MARI SATO
}

Department of Biochemistry and Molecular Biology, Graduate School of Dental Medicine, Hokkaido University, Kita-ku, Sapporo 060-8586, Japan

Received April 11, 2013; Accepted September 16, 2013

DOI: $10.3892 / \mathrm{mmr} .2013 .1713$

\begin{abstract}
The canonical Wnt signaling pathway is crucial for the regulation of bone mass in humans and for the development of osteoblasts. MicroRNAs (miRs) represent a class of non-coding RNAs, 22 nucleotides in length, that regulate gene expression by targeting mRNAs for cleavage or translational repression. Several previous studies have demonstrated the involvement of miRNAs in modulating gene expression in osteoblasts and regulating osteoblast differentiation. In the present study, microRNA profiling was conducted using Wnt3a-C2C12 cells; C2C12 cells were transfected with a Wnt3a expression plasmid to activate canonical Wnt signaling. miR-34b-5p and miR-34c were identified to be upregulated by the activation of canonical Wnt signaling in $\mathrm{C} 2 \mathrm{C} 12$ cells. Expression of mature $\mathrm{miR}-34 \mathrm{~b} / \mathrm{c}$ increased from low levels at day 0 to maximum levels at day 28 of MC3T3-E1 cell differentiation. To analyze the effects of these miRNAs on osteoblast differentiation, an antisense inhibitor was transfected into MC3T3-E1 cells and osteoblast-related gene expression was investigated. Knockdown of miR34b/c enhanced osteocalcin mRNA expression; however, alkaline phosphatase mRNA expression and activity were decreased by $\mathrm{miR} 34 \mathrm{~b} / \mathrm{c}$ inhibition. These results indicated that $\mathrm{miR}-34 \mathrm{~b} / \mathrm{c}$ regulates gene expression by targeting regulators of the osteogenic pathways and thereby contributes to osteoblast differentiation.
\end{abstract}

\section{Introduction}

Bone formation is a complex process that involves the recruitment of progenitor cells, proliferation and differentiation of these cells into osteoblasts, and the resultant secretion of abundant bone extracellular matrix proteins that coordinates

Correspondence to: Professor Masato Tamura, Department of Biochemistry and Molecular Biology, Graduate School of Dental Medicine, Hokkaido University, North 13 West 7, Kita-ku, Sapporo 060-8586, Japan

E-mail:mtamura@den.hokudai.ac.jp

Key words: miRNA, Wnt, osteoblasts, differentiation, osteocalcin the mineralization process $(1,2)$. Differentiation into cells of the osteoblast lineage is dependent upon multiple extracellular cues received by the cell, and involves complex pathways regulated at the transcriptional and posttranscriptional levels. However, the regulation of these pathways is not fully understood. Bone-anabolic agents, such as bone morphogenetic protein (BMP)-2, parathyroid hormone and canonical Wnts promote osteoblast differentiation by stimulating distinct second messenger pathways (3). The canonical Wnt signaling pathway is crucial for the regulation of bone formation and osteoblastic differentiation in humans $(4,5)$. In addition, we have previously demonstrated that canonical Wnt signaling induces the expression of osteoprotegerin (6).

MicroRNAs (miRNAs) are small non-coding RNAs that are 22 nucleotides in length. One strand of this short-lived duplex is degraded by an unknown nuclease, while the other strand is selected and incorporated into the effector complex termed the RNA-induced silencing complex (RISC). RISC interacts with the mRNA target in a sequence-specific manner and regulates specific target genes by repressing their translation and/or promoting the degradation of their transcribed mRNAs by binding to their 3'-untranslated regions (7-9). More than 1,000 miRNAs have been discovered in mammals, a number of which are expressed in a tissue-specific manner (10). An accumulating number of studies have suggested that the regulation of cell differentiation by miRNAs is a notable component of the regulatory machinery. In a previous study, striated-muscle-specific miR-206 was shown to be downregulated by BMP-2 at the post-transcriptional level in $\mathrm{C} 2 \mathrm{C} 12$ cells (11). In addition, several studies have demonstrated that certain miRNAs act as important orchestrators of osteoblast-specific genes that are required for osteoblast differentiation, acting through their regulation of diverse signaling molecules and pathways (12-19).

In the present study, microRNA profiling studies were conducted during the induction of osteoblast differentiation by canonical Wnt signaling. miR-34b-5p and miR-34c were identified to be upregulated by the activation of canonical Wnt signaling in $\mathrm{C} 2 \mathrm{C} 12$ cells. Thus, the present study also investigated the involvement of miR-34b/c throughout osteoblast differentiation. During the maturation of preosteoblasts, such as MC3T3-E1 cells, these miRNAs are upregulated at the mineralization stage. These results indicated that $\mathrm{miR}-34 \mathrm{~b} / \mathrm{c}$ 
regulated osteoblast differentiation, controlling the expression of osteocalcin and alkaline phosphatase (ALP).

\section{Materials and methods}

Cell cultures. The $\mathrm{C} 2 \mathrm{C} 12$ mouse myoblast cell line and the MC3T3-E1 mouse calvarial osteoblast cell line were obtained as described previously (20). The Kusa-O and ST-2 mouse mesenchymal cell lines were obtained from the RIKEN Cell Bank (Tsukuba, Japan). Wnt3a-C2C12 cells were established as described previously (20). Cells were cultured in $\alpha$-minimum essential medium (MEM) supplemented with $10 \%$ fetal bovine serum (SAFC Biosciences, Inc., Lenexa, KS, USA) at $37^{\circ} \mathrm{C}$ in a $5 \% \mathrm{CO}_{2}$ humidified atmosphere. For osteoblastic differentiation, the cells were cultured in $\alpha$-MEM containing $5 \mathrm{mM}$ $\beta$-glycerophosphate, $100 \mu \mathrm{g} / \mathrm{ml}$ ascorbic acid and $10^{-8} \mathrm{M}$ dexamethasone (Sigma-Aldrich, St. Louis, MO, USA) for 1-3 weeks. The culture medium was replaced every 3 days.

miRNA microarray and data analysis. miRNA was extracted from the cells using the mirVana miRNA isolation kit (Ambion, Austin, TX, USA). Labeled miRNAs were hybridized onto 3D-Gene miRNA oligo chips containing $>700$ antisense probes (Toray Industries Inc., Tokyo, Japan). The microarray images obtained were analyzed using Genepix Pro ${ }^{\mathrm{TM}} 4.0$ software (Molecular Devices, Sunnyvale, CA, USA). Differences in the total fluorescence intensity between arrays were adjusted by global normalization. The mean values for duplicate microarrays were calculated and used for comparison between the groups. When the difference in relative miRNA expression between the two groups was $>2.0$-fold, this was defined as a change in the expression.

Transfection assay. The antisense inhibitors were designed to bind to specific endogenous miRNAs and inhibit their activities when introduced into cells. The following 5'- and 3'-phosphorylated RNAs with full 2'-O-methyl modifications were chemically synthesized by Nippon Bio Services Co. Ltd. (Asaka, Japan): 5' - ACA AUCAGCUA AUUACACUGCCU-3' for antimiR-34b5p; 5'-GCAAUCAGCUAACUACAC UGCCU-3' for antimiR-34c; and 5'-UCAGCAGCACAGUCA AUACUGG-3' for antimiR-16. The antimiRNAs were transfected into $\mathrm{C} 2 \mathrm{C} 12$ cells using lipofectamine 2000 cationic liposomes (Invitrogen Life Technologies, Carlsbad, CA, USA) according to the manufacturer's instructions and were then cultured for 2 days. AntimiR-16 served as a control for nonspecific effects.

Quantitation of miRNA expression. To analyze the miRNA level, RNA was extracted from the cells using a mirVana miRNA isolation kit (Ambion) and cDNA was synthesized with specific miRNA primers from the TaqMan MicroRNA assay (Applied Biosystems, Carlsbad, CA, USA). The resulting cDNA was amplified by PCR using the TaqMan MicroRNA assay system with the StepOne ${ }^{\circledR}$ Real Time PCR system (Applied Biosystems) (21). The relative expression level of miRNA was quantified using the comparative $\mathrm{C}_{T}$ method with sno234 RNA as the endogenous control.

Quantitation of mRNA expression by quantitative polymerase chain reaction $(q P C R)$. To analyze the mRNA expression

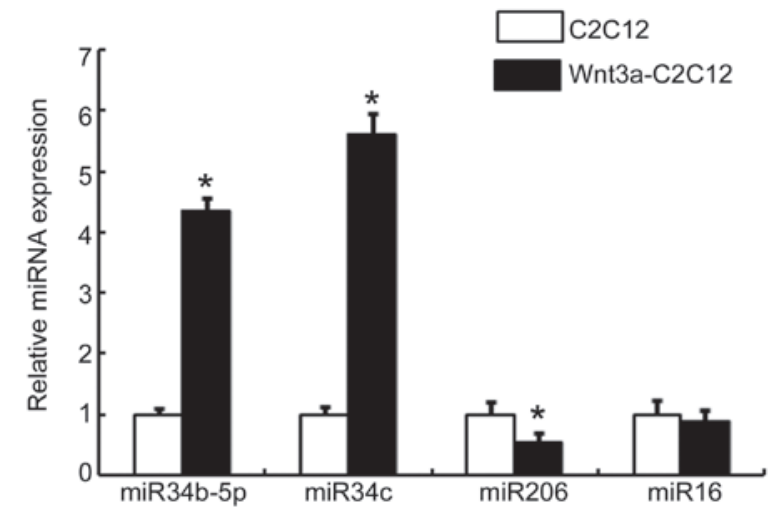

Figure 1. Regulation of miR-34b/c expression by canonical Wnt signaling. $\mathrm{C} 2 \mathrm{C} 12$ and Wnt3a-C2C12 cells were harvested at confluence and total RNA was extracted. Expression of miR-34b-5p, miR-34c, miR-206 and miR-16 was then analyzed by qPCR and expression levels were normalized against those of sno234 RNA. ${ }^{*} \mathrm{P}<0.05$ as determined by a t-test for paired data.

level, qPCR was performed using assay-on-demand TaqMan probes (Applied Biosystems) with the StepOne ${ }^{\circledR}$ real time PCR system (Applied Biosystems). The relative gene expression level was quantified using the comparative $\mathrm{C}_{T}$ method with glyceraldehyde 3-phosphate dehydrogenase as the endogenous control.

ALP activity and ALP staining. Cells were washed twice with phosphate-buffered saline, $200 \mu 1$ lysis buffer was added to the cell layer and the cells were incubated on ice for $5 \mathrm{~min}$. The cell lysate was then sonicated for 1 min (Virtis Virsonic; Virtis Co., Gardiner, NY, USA) and centrifuged at 1,000 $\times \mathrm{g}$ and $4^{\circ} \mathrm{C}$ for $10 \mathrm{~min}$ (MX-300; Tomy, Tokyo, Japan). ALP activity was assayed by a spectrophotometric method using a LabAssay ${ }^{\mathrm{TM}}$ ALP kit (Wako Pure Chemical Industries Ltd., Osaka, Japan). The absorbance of each well at $405 \mathrm{~nm}$ was measured with a microplate reader (iMark; Bio-Rad, Richmond, CA, USA).

Statistical analysis. All experiments were repeated three to four times and representative results are shown. The data are presented as the mean \pm SD, and were analyzed by Student's t-test. $\mathrm{P}<0.05$ was considered to indicate a statistically significant difference.

\section{Results}

Expression of miRNAs in Wnt3a-C2C12 cells. $\mathrm{C} 2 \mathrm{C} 12$ is a multipotent cell line that provides a well-characterized model system and has been demonstrated to differentiate into myotubes and osteoblasts. To investigate the involvement of canonical Wnt signaling in osteoblastic differentiation, we previously generated a $\mathrm{C} 2 \mathrm{C} 12$ cell line stably expressing Wnt3a (20). C2C12 cells were transfected with the Wnt3a expression plasmid to activate canonical Wnt signaling and were then selected using G418 to establish a stable cell line, termed Wnt3a-C2C12 (20). To confirm the activation of canonical Wnt signaling in these cells, the reporter plasmid that carries six tandem repeats of the Lef 1/Tcf binding site was transfected with Topflash, which demonstrated that the 
$\mathbf{A}$

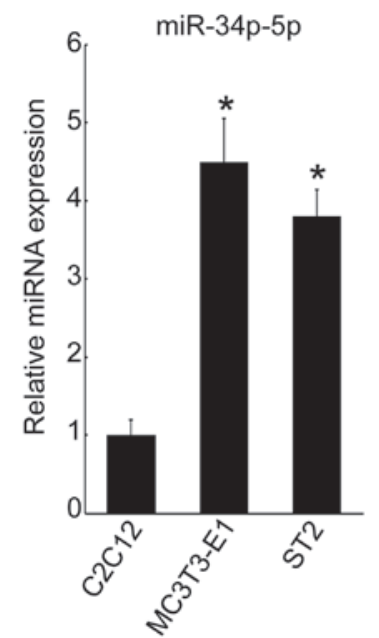

B

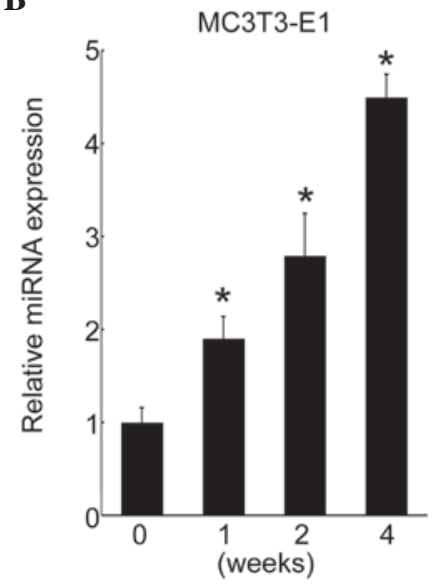

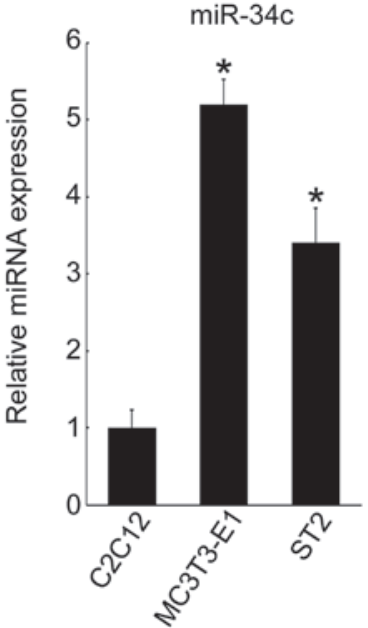

Kusa-O

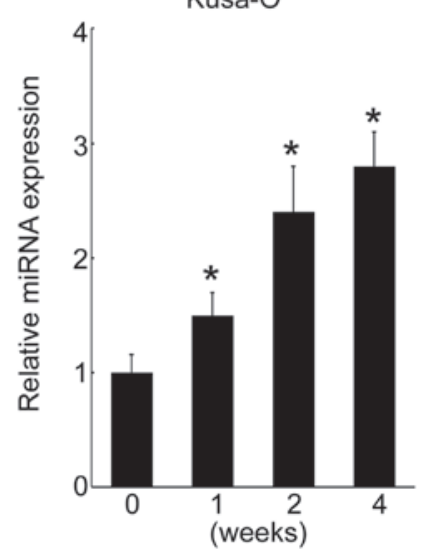

Figure 2. Expression of miR-34b/c in osteoblasts and their regulation during osteoblastic differentiation. (A) C2C12, MC3T3-E1 and ST-2 cells were harvested at confluence, and total RNA was extracted. (B) MC3T3-E1 or Kusa-O cells were cultured with $10 \%$ fetal bovine serum in $\alpha$-minimum essential medium containing $5 \mathrm{mM} \beta$-glycerophosphate, $100 \mu \mathrm{g} / \mathrm{ml}$ ascorbic acid and $10^{-8} \mathrm{M}$ dexamethasone for the indicated times. Total RNA was extracted and the expression levels of miR-34b-5p (A) and miR-34c (A and B) were analyzed by qPCR. Expression levels were normalized against those of sno234 RNA. "P<0.05 compared with control, as determined by a t-test for paired data.

promoter activity of Topflash is enhanced in Wnt3a-C2C12 cells. Previously, ALP activity was observed, which indicated that canonical Wnt signaling is active in these cells (20). Therefore, in the present study, total RNA was extracted from Wnt3a-C2C12 and $\mathrm{C} 2 \mathrm{C} 12$ cells, and the expression of miRNAs was analyzed using an established microarray platform to determine the potential involvement of miRNAs in canonical Wnt signaling-mediated osteoblastic differentiation. From the miRNAs that were expressed at significant levels and showed changes in expression in response to Wnt signaling, five miRNAs were identified, which were upregulated in Wnt3a-C2C12 cells compared with $\mathrm{C} 2 \mathrm{C} 12$ cells. Two of the miRNAs were downregulated by Wnt3a stimulation, while in the upregulated group, miR-34b-5p, miR-34b-3p and miR-34c were among the most markedly changed. These changes in expression of miR-34b-5p and miR-34c in Wnt3a-C2C12 and $\mathrm{C} 2 \mathrm{C} 12$ cells were confirmed by qPCR. As expected, expression of miR-34b-5p and miR-34c was upregulated in Wnt3a-C2C12 cells compared with that in the vehicle-transfected $\mathrm{C} 2 \mathrm{C} 12$ cells (Fig. 1). No regulation of non-specific miR-16 expression was observed in the Wnt3a-C2C12 cells. However, miR-206 was highly expressed in the $\mathrm{C} 2 \mathrm{C} 12$ cells, whereas its expression was lower in the Wnt3a-C2C12 cells (Fig. 1). These results indicated that the canonical Wnt signaling pathway may be involved in regulating the expression of miR-34b-5p and $\mathrm{miR}-34 \mathrm{c}$.

Cell type-specific expression of miR-34b-5p and $m i R-34 c$. The cell type-specific expression of miR-34b-5p and miR-34c was investigated in a myoblastic cell line and two osteoblastic cell lines. In MC3T3-E1 and ST-2 osteoblastic cells, miR-34b-5p and miR-34c were highly expressed compared with that in myoblastic $\mathrm{C} 2 \mathrm{C} 12$ cells (Fig. 2A). By contrast, miR-16 expression levels were not altered (data not shown). MC3T3-E1 cells derived from fetal mouse calvaria undergo maturation when cultured in osteogenic medium. In the present study, it was demonstrated that the expression of miR-34b-5p and miR-34c was induced by canonical Wnt signaling, which suggests that these miRNAs may act as stimulators of osteoblastic differentiation. To understand the involvement of miR-34b/c expression in osteoblastic differentiation, the effect of cell culture on the level of miRNA expression over time was investigated. Expression of miR-34c increased over the culture period from day 0 and reached maximum levels at four weeks in MC3T3-E1 cells (Fig. 2B). This pattern of expression of miRNA was confirmed in the Kusa-O mouse mesenchymal 
A

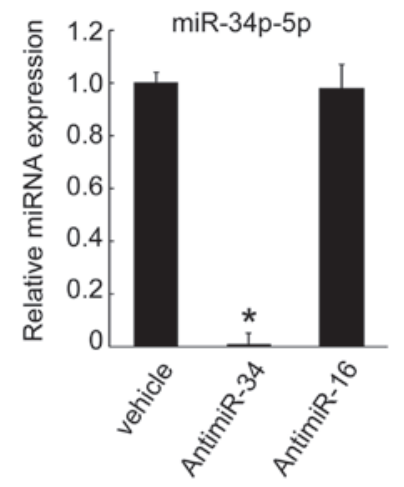

B

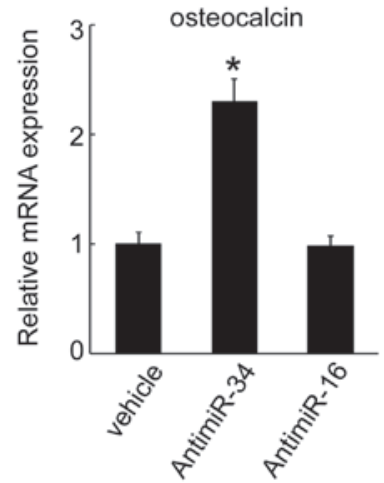

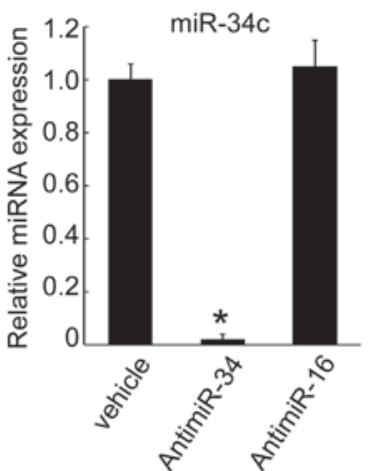

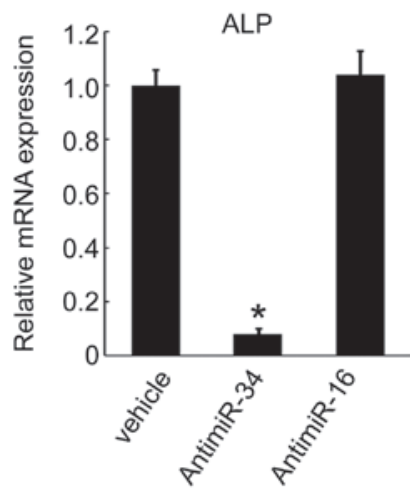

C

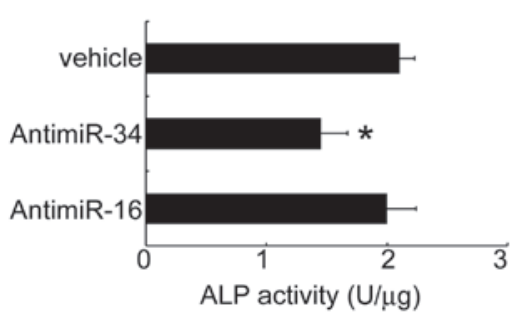

Figure 3. Effects of inhibition of miR-34b/c expression on osteocalcin and alkaline phosphatase (ALP) mRNA expression in MC3T3-E1 cells. MC3T3-E1 cells were cultured with $10 \%$ fetal bovine serum in $\alpha$-minumum essential medium. Following overnight incubation, the cells were transiently transfected with antimiR-34c or antimiR-16 $(1 \mu \mathrm{M})$ and incubated again overnight. The culture medium was then replaced with fresh medium and the cells were cultured for a further $24 \mathrm{~h}$. (A) Total RNA was extracted and the expression levels of miR-34b-5p and miR-34c was analyzed by quantitative polymerase chain reaction. Expression levels were normalized against those of sno234 RNA. (B) qPCR was performed to quantify the expression of osteocalcin and ALP mRNA. Expression levels were normalized against those of glyceraldehyde 3 -phosphate mRNA. (C) The mean ALP activity of the cells. * $<0.05$, compared with vehicle as determined by a t-test for paired data.

cell line, with an increase up to four weeks when proliferation ceased in the multilayered cell nodules (Fig. 2B). These results indicated that miR-34b/c was induced during osteoblastic differentiation in vitro.

Expression of osteoblastic genes following transfection with anti-miRNA. To further analyze the function of miRNAs in osteoblastic differentiation, anti-miRNA was transfected and the expression of osteoblastic genes was measured. Following the transfection of MC3T3-E1 cells with anti-miR34c, the expression levels of miR-34b-5p and miR-34c significantly diminished to undetectable levels (Fig. 3A). In these cells, the expression of specific genes that characterize osteoblastic differentiation (such as osteocalcin) increased, whereas the expression levels of ALP mRNA were reduced by miR34 inhibition (Fig. 3B). In addition, ALP activity declined following knockdown of miR-34 in MC3T3-E1 cells (Fig. 3C), supporting the hypothesis that miR-34 regulates osteoblastic gene expression.

\section{Discussion}

To date, several studies have demonstrated a regulatory role of miRNAs in osteoblast differentiation (12-19). Certain miRNAs have also been shown to act as negative regulators of osteoblast differentiation, such as miR-26a and miR-125b. In the present study a novel miRNA that regulates canonical Wnt signaling was investigated. Canonical Wnt signaling induces $\mathrm{miR}-34 \mathrm{~b} / \mathrm{c}$ expression.

Three miR-34s have been identified, miR-34b and miR-34c are encoded by a gene located on mouse chromosome 9, and miR-34a is encoded by a gene located on mouse chromosome 4. miR-34b-5p and miR-34b-3p are transcribed in tandem, in the same primary miRNA (22). In general, miRNAs are transcribed by RNA polymerase II or III, and transcription is regulated by the interaction of trans-acting factors with the promoter region (23). According to the current model of canonical Wnt action, glycogen synthase kinase (GSK)-3 $\beta$ phosphorylates $\beta$-catenin and 
thereby induces rapid degradation of $\beta$-catenin in cells that lack Wnt signaling. Wnt inhibits GSK-3 $\beta$, thus stabilizing $\beta$-catenin, which interacts with several molecules in the cytosol, including lymphoid enhancer factor $1 / \mathrm{T}$ cell factor (Lef1/Tcf). A complex involving the transcription factor Lef1/Tcf and $\beta$-catenin regulates the expression of several target genes via Lef1/Tcf binding sites within gene promoter regions (24). The results suggested that the miR-34b/c gene promoter region may contain an Lef1/Tcf binding site that responds to canonical Wnt signaling.

A previous study indicated that human miR-29a expression is induced by canonical Wnt signaling and that miR-29a subsequently downregulates the Wnt signaling antagonists Dkk1, Kremen2 and sFRP2, potentiating Wnt signaling (13). These two actions promote a gene expression program that is required for osteoblast differentiation. In the present study, miR-34b/c and miR-29a expression increased in Wnt3a-C2C12 cells, as shown by microarray analysis (data not shown). However the correlation between miR-34b/c and miR-29a is not clear when canonical Wnt signaling is activated in osteoblasts. An advantage of using multiple miRNAs may be the provision of a variety of options for biological regulation in cells.

It has been demonstrated that p53 transactivates miR-34, which then suppresses Lef1/Tcf complexes by targeting the canonical Wnt pathway (25). Furthermore, Wei et al (26) demonstrated that $\mathrm{miR} 34 \mathrm{~b} / \mathrm{c}$ affects skeletogenesis during embryonic development and influences bone mass accrual following birth. It was suggetsed that two molecular mechanisms may be involved. Initially, miR-34b/c inhibits the osteoblast proliferation by suppressing CyclinD1, CDK4 and CDK6 accumulation, and miR34b/c then inhibits terminal differentiation of osteoblasts through the inhibition of SATB2, a nuclear matrix protein (26). Similar to the results of the present study, Wei et al demonstrated that osteocalcin expression was increased in miR-34-/- osteoblasts. In our study, inhibition of miR34b/c downregulated the expression of ALP, which is a marker of osteoblastic differentiation. The exact mechanisms by which $\mathrm{miR}-34 \mathrm{~b} / \mathrm{c}$ regulates gene expression or its function during osteoblastic differentiation remains to be elucidated. miRNAs provide a mechanism for fine-tuning intricate cellular processes. In combination with a variety of signaling molecules and transcription factors, miRNAs may aid in controlling the complex program of osteoblastic differentiation.

The present study supports the hypothesis that canonical Wnt signaling regulates $\mathrm{miR}-34 \mathrm{~b} / \mathrm{c}$ expression and in turn regulates gene expression by altering cellular function and/or signaling activities, thus controlling osteoblastic differentiation in bone tissue. This regulatory circuit provides additional insight into the manner in which canonical Wnt signaling modulates the expression of miRNAs during osteoblast differentiation and may be of value in the future, aiding in the development of novel therapies to improve bone mass in patients with bone disorders.

\section{Acknowledgements}

This study was supported in part by the Japan Ministry of Education, Culture, Sports, Science and Technology Grants-in-aid (grant no. 22390346) (MT).

\section{References}

1. Young MF: Bone matrix proteins: more than markers. Calcif Tissue Int 72: 2-4, 2003

2. Ortega N, Behonick DJ and Werb Z: Matrix remodeling during endochondral ossification. Trends Cell Biol 14: 86-93, 2004.

3. Canalis E: Update in new anabolic therapies for osteoporosis. J Clin Endocrinol Metab 95: 1496-1504, 2010.

4. Krishnan V, Bryant H and Macdougald OA: Regulation of bone mass by Wnt signaling. J Clin Invest 116: 1202-1209, 2006.

5. Tamura M, Nemoto E, Sato MM, Nakashima A and Shimauchi $\mathrm{H}$ : Role of the Wnt signaling pathway in bone and tooth. Front Biosci (Elite Ed) 2: 1405-1413, 2010.

6. Sato M, Nakashima A, Nashimoto M, Yawaka Y and Tamura M: Bone morphogenetic protein-2 enhances Wnt/beta-catenin signaling-induced osteoprotegerin expression. Genes Cells 14: 141-153, 2009.

7. Filipowicz W, Bhattacharyya S and Sonenberg N: Mechanisms of post-transcriptional regulation by microRNAs: are the answers in sight? Nat Rev Genet 9: 102-114, 2008.

8. Ding XC, Weiler J and Grosshans H: Regulating the regulators: mechanisms controlling the maturation of microRNAs. Trends Biotechnol 27, 27-36, 2009.

9. Sayed D and Abdellatif M: MicroRNAs in development and disease. Physiol Rev 91: 827-887, 2011.

10. Williams AE: Functional aspects of animal microRNAs. Cell Mol Life Sci 65: 545-562, 2008.

11. Sato M, Nashimoto M, Katagiri T, Yawaka Y and Tamura M: Bone morphogenetic protein-2 down-regulates miR-206 expression by blocking its maturation process. Biochem Biophys Res Commun 383: 125-129, 2009.

12. Kapinas K, Kessler CB and Delany AM: miR-29 suppression of osteonectin in osteoblasts: regulation during differentiation and by canonical Wnt signaling. J Cell Biochem 108: 216-224, 2009.

13. Kapinas K, Kessler C, Ricks T, Gronowicz G and Delany AM miR-29 modulates Wnt signaling in human osteoblasts through a positive feedback loop. J Biol Chem 285: 25221-25231, 2010.

14. Schoolmeesters A, Eklund T, Leake D, et al: Functional profiling reveals critical role for miRNA in differentiation of human mesenchymal stem cells. PLoS One 4: e5605, 2009.

15. Itoh T, Nozawa Y and Akao Y: MicroRNA-141 and -200a are involved in bone morphogenetic protein-2-induced mouse pre-osteoblast differentiation by targeting distal-less homeobox 5 . J Biol Chem 284: 19272-19279, 2009.

16. Hu R, Liu W, Li H, et al: A Runx2/miR-3960/miR-2861 regulatory feedback loop during mouse osteoblast differentiation. J Biol Chem 286: 12328-12339, 2011.

17. Hassan MQ, Gordon JA, Beloti MM, et al: A network connecting Runx2, SATB2, and the miR-23a 27a 24-2 cluster regulates the osteoblast differentiation program. Proc Natl Acad Sci USA 107: 19879-19884, 2010.

18. Eskildsen T, Taipaleenmäki H, Stenvang J, et al: MicroRNA-138 regulates osteogenic differentiation of human stromal (mesenchymal) stem cells in vivo. Proc Natl Acad Sci USA 108: 6139-6144, 2011.

19. Zhang Y, Xie RL, Croce CM, et al: A program of microRNAs controls osteogenic lineage progression by targeting transcription factor Runx2. Proc Natl Acad Sci USA 108: 9863-9868, 2011.

20. Nakashima A, Katagiri T and Tamura M: Cross-talk between Wnt and bone morphogenetic protein 2 (BMP-2) signaling in differentiation pathway of C2C12 myoblasts. J Biol Chem 280: 37660-37668, 2005.

21. Uyama M, Sato MM, Kawanami M and Tamura M: Regulation of osteoblastic differentiation by the proteasome inhibitor bortezomib. Genes Cells 17: 548-558, 2012.

22. Landgraf $\mathrm{P}$, Rusu M, Sheridan R, et al: A mammalian microRNA expression atlas based on small RNA library sequencing. Cell 29: 1401-1414, 2007.

23. Lee Y, Kim M, Han J, et al: MicroRNA genes are transcribed by RNA polymerase II. EMBO J 23: 4051-4060, 2004.

24. Yochum G, McWeeney S, Rajaraman V, Cleland R, Peters S and Goodman RH: Serial analysis of chromatin occupancy identifies beta-catenin target genes in colorectal carcinoma cells. Proc Natl Acad Sci USA 104: 3324-3329, 2007.

25. Kim NH, Kim HS, Kim NG, et al: p53 and microRNA-34 are suppressors of canonical Wnt signaling. Sci Signal 4: ra71, 2011.

26. Wei J, Shi Y, Zheng L, et al: miR-34s inhibit osteoblast proliferation and differentiation in the mouse by targeting SATB2. J Cell Biol 197: 509-521, 2012. 